\title{
A 2.5D BEM-FEM USING A SPECTRAL APPROACH TO STUDY SCATTERED WAVES IN FLUID-SOLID INTERACTION PROBLEMS
}

\author{
FRANCISCO JAVIER CRUZ-MUÑOZ ${ }^{1}$, ANTONIO ROMERO ${ }^{1}$, PEDRO GALVÍN $^{1}$ \& ANTÓNIO TADEU ${ }^{2,3}$ \\ ${ }^{1}$ Departamento de Mecánica de Medios Continuos y Teoría de Estructuras, Escuela Técnica Superior de Ingeniería, \\ Universidad de Sevilla, Spain \\ ${ }^{2}$ ITeCons - Institute for Research and Technological Development in Construction, Energy, Environment and \\ Sustainability, Portugal \\ ${ }^{3}$ ADAI - LAETA, Department of Civil Engineering, University of Coimbra, Portugal
}

\begin{abstract}
This work presents a two-and-a-half dimensional (2.5D) spectral formulation based on the finite element method (FEM) and the boundary element method (BEM) to study wave propagation in acoustic and elastic waveguides. The analysis involved superposing two dimensional (2D) problems with different longitudinal wavenumbers. A spectral finite element (SFEM) is proposed to represent waveguides in solids with arbitrary cross-section. Moreover, the BEM is extended to its spectral formulation (SBEM) to study unbounded fluid media and acoustic enclosures. Both approaches use Lagrange polynomials as element shape functions at the Legendre-Gauss-Lobatto (LGL) points. The fluid and solid subdomains are coupled by applying the appropriate boundary conditions at the limiting interface. The proposed method is verified by means of a benchmark problem regarding the scattering of waves by an elastic inclusion. The convergence and the computational effort are evaluated for different $h-p$ strategies. Numerical results show good agreement with the reference solution. Finally, the proposed method is used to study the pressure field generated by an array of elastic fluid-filled scatterers immersed in an acoustic medium.
\end{abstract}

Keywords: SBEM, SFEM, fluid-solid interaction, waveguide, scattered waves, two-and-a-half dimension.

\section{INTRODUCTION}

Many engineering fields involve time harmonic wave propagation, such as fluid acoustics and solid scattering. Hybrid methods based on the boundary element method (BEM) and the finite element method (FEM) are suitable for studying solid and fluid interaction in unbounded media. Both methods have been used in various works to predict the response of coupled fluid-structure problems. For the low frequency range, the standard formulation using linear elements accurately represents the fluid and solid scattering waves. However, at high frequencies, the solution deteriorates due to so-called pollution effects [1], [2]. Higher element approximations are needed to obtain accurate results.

Different approaches have been proposed in the past to overcome the pollution effect in two and three dimensional problems [3]. The $h$-refinement method has proved to be useless at high wavenumbers because of the necessary computational effort to represent small wavelengths [4]. Many works suggest using high-order elements to improve the convergence rate of standard formulations. The boundary and finite element methods have thus been extended to their spectral formulations [5], [6] approximating the field variables by high-order interpolation shape functions. Such functions are polynomials over non-uniformly spaced nodes to avoid the Runge phenomenon.

In this work, we propose a two-and-a-half dimensional coupled spectral formulation based on the FEM and the BEM to study wave propagation in fluid and solid waveguides. The proposed method is useful for problems whose geometry and material properties are both uniform in one direction, whereas the excitation exhibits a three dimensional distribution. 


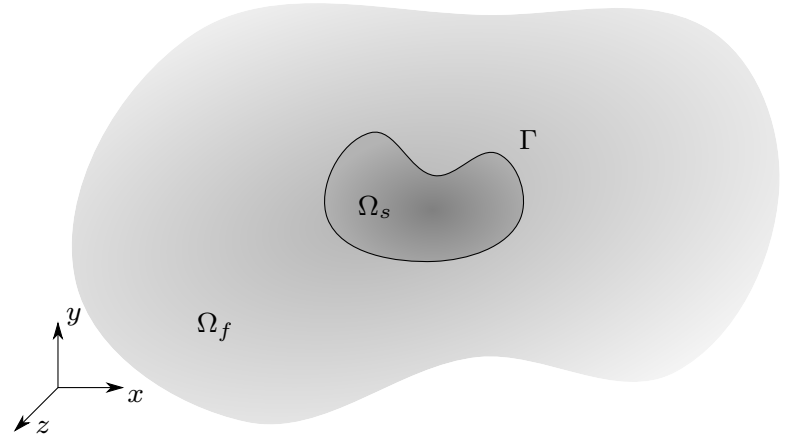

Figure 1: Donnain decomposition:

The spectral approach allows the analysis of wave propagation at high wavenumbers, which The spectral approach atlows the analysis of wave propagation at high wavenumbers, which redufes the pollutipn effect with low computational effort.

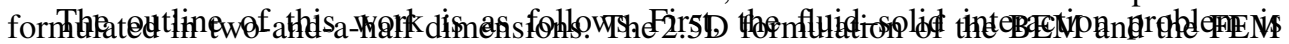

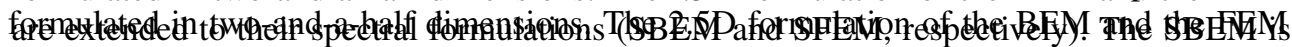

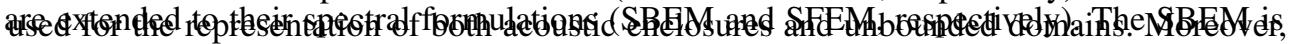

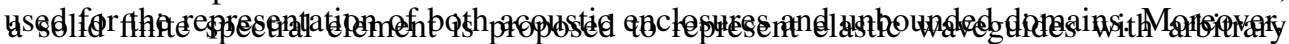

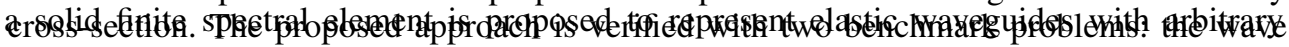

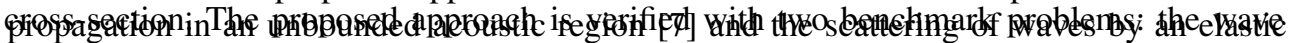

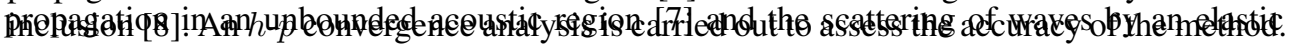

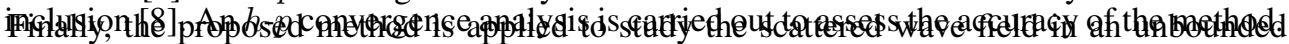

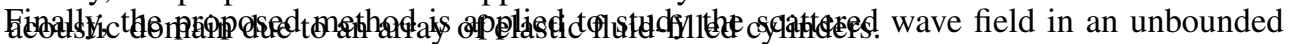
acoustic domain due to an array of elastic fluid-filled cylinders.

The spectral formulations of the BEMERICAEMOREELeveloped to represent an elastic

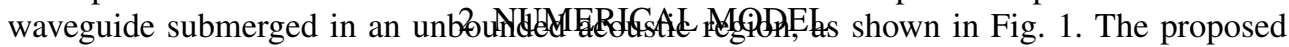

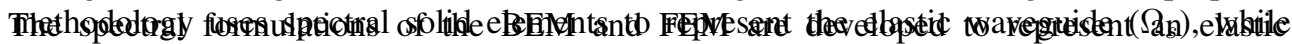

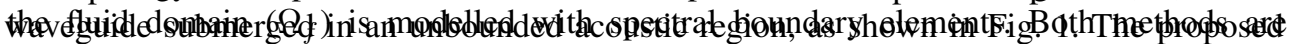

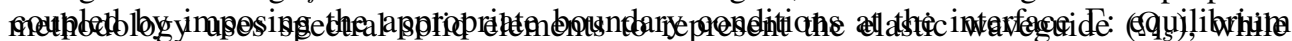

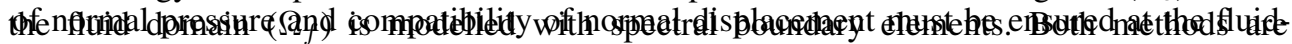

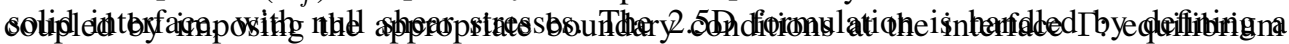

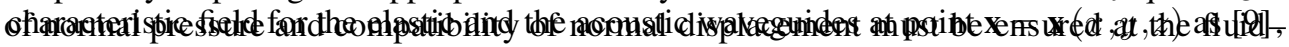
$\$ d \mathrm{Adl}$ interface, with null shear stresses. The 2.5D formulation is handled by defining a

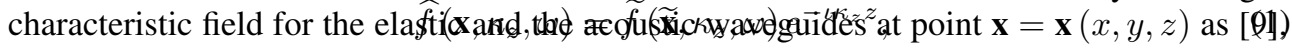
[10]:

where $\widetilde{f}\left(\widetilde{\mathbf{x}}, \kappa_{z}, \omega\right)$ is the frequency-wavenumber representation of an unknown variable

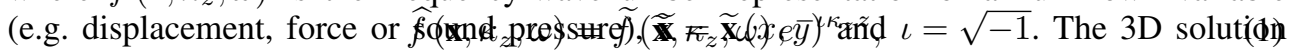
is computed as the superposition of $2 \mathrm{D}$ solutions with different wavenumber $\kappa_{z}$ :

where $\widetilde{f}\left(\widetilde{\mathbf{x}}, \kappa_{z}, \omega\right)$ is the frequency-yabenumber representation of an unknown variable

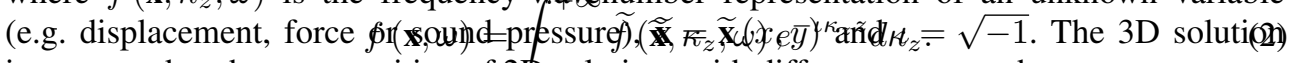
is computed as the superposition of $2 \mathrm{D}$-solutions with different wavenumber $\kappa_{z}$ :

\subsection{Spectral boundary element method (SBEM)}

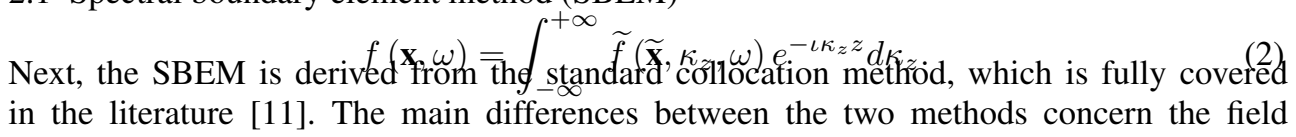




\subsection{Spectral boundary element method (SBEM)}

Next, the SBEM is derived from the standard collocation method, which is fully covered in the literature [11]. The main differences between the two methods concern the field variable approximation and the proposed element integration scheme. In both cases, the boundary integral representation is derived from the Helmholtz equation [11], considering a homogeneous fluid domain denoted by its volume $\Omega_{f}$ and boundary $\Gamma$. Although the proposed method is used to obtain the three-dimensional radiated field by an elastic waveguide with an arbitrary cross section, the problem is computed as the superposition of 2D problems with different wavenumber $\kappa_{z}$. Therefore, the boundary $\Gamma$ is reduced to its cross section $\Sigma$ at the coordinate plane $z=0$. Thus, the integral representation of the fluid pressure for a point $\widetilde{\mathbf{x}}_{i}$ located at the boundary $\Gamma$ in the frequency-wavenumber domain is:

$$
\begin{aligned}
& c_{i}\left(\widetilde{\mathbf{x}}_{i}\right) \widetilde{p}_{i}\left(\widetilde{\mathbf{x}}_{i}, \kappa_{z}, \omega\right) \\
& \quad=-\int_{\Sigma}\left(\iota \rho \omega^{2} \widetilde{u}_{i}\left(\widetilde{\mathbf{x}}_{i}, \kappa_{z}, \omega\right) \widetilde{\Psi}\left(\widetilde{\mathbf{x}}, \kappa_{z}, \omega ; \widetilde{\mathbf{x}}_{i}\right)+\widetilde{p}_{i}\left(\widetilde{\mathbf{x}}_{i}, \kappa_{z}, \omega\right) \frac{\partial \widetilde{\Psi}\left(\widetilde{\mathbf{x}}, \kappa_{z}, \omega ; \widetilde{\mathbf{x}}_{i}\right)}{\partial \mathbf{n}}\right) d \Sigma,
\end{aligned}
$$

where $\widetilde{p}\left(\widetilde{\mathbf{x}}_{i}, \kappa_{z}, \omega\right)$ and $\widetilde{u}\left(\widetilde{\mathbf{x}}_{i}, \kappa_{z}, \omega\right)$ are the sound pressure and the particle normal displacement at the cross section $\Sigma$ of the boundary $\Gamma$, respectively. $\widetilde{\Psi}\left(\widetilde{\mathbf{x}}, \kappa_{z}, \omega ; \widetilde{\mathbf{x}}_{i}\right)$ is the solution to the Helmholtz equation at point $\widetilde{\mathbf{x}}$ due to a point source located at $\widetilde{\mathbf{x}}_{i}$ [12]. The integral-free term $c_{i}\left(\widetilde{\mathbf{x}}_{i}\right)$ only depends on the boundary geometry at the collocation point $\widetilde{\mathbf{x}}_{i}$.

The boundary is discretised into $N$ elements, with $\Sigma=\bigcup_{j=1}^{N} \Sigma^{j}$, and the field variables are approximated within the element using a set of interpolation shape functions $\phi(\xi)$. The proposed methodology uses Lagrange polynomials of order $p$ as interpolation shape functions given by:

$$
\phi^{k}=\prod_{j \neq i} \frac{\xi-\xi_{i}}{\xi_{j}-\xi_{i}},
$$

where nodal coordinates $\xi_{i}$ are found at the Lobatto-Gauss-Legendre (LGL) points. The use of a family of orthogonal polynomial $\left(\phi^{i}\left(\xi_{j}\right)=\delta_{i j}\right)$, such as Lagrange polynomials, as interpolation shape functions, mitigates the appearance of the Runge phenomenon, which can result in a highly ill-conditioned problem for high-order elements [13].

Once the sound pressure and particle normal displacement are approximated within the element, the former integrals in eqn (3) become:

$$
\begin{aligned}
\widetilde{g}_{i}^{j}\left(\widetilde{\mathbf{x}}, \kappa_{z}, \omega ; \widetilde{\mathbf{x}}_{i}\right) & =\int_{\Sigma^{j}} \phi \widetilde{\Psi}\left(\widetilde{\mathbf{x}}, \kappa_{z}, \omega ; \widetilde{\mathbf{x}}_{i}\right) d \Sigma, \\
\widetilde{h}_{i}^{j}\left(\widetilde{\mathbf{x}}, \kappa_{z}, \omega ; \widetilde{\mathbf{x}}_{i}\right) & =\int_{\Sigma^{j}} \phi \frac{\partial \widetilde{\Psi}\left(\widetilde{\mathbf{x}}, \kappa_{z}, \omega ; \widetilde{\mathbf{x}}_{i}\right)}{\partial n} d \Sigma .
\end{aligned}
$$

The spatial integrations in eqns (5) and (6) are numerically evaluated using a standard GaussLegendre quadrature with $(p+1)$ integration points whenever the collocation point does not belong to the integration element. However, the integrals become singular when the collocation point falls on the integration element. These integrals are numerically evaluated 
by element subdivision, isolating the collocation point at the integration endpoints

$$
\begin{aligned}
& \int_{\Sigma^{j}} \tilde{f}\left(\widetilde{\mathbf{x}}, \kappa_{z}, \omega ; \widetilde{\mathbf{x}}_{i}\right) d \Sigma=\int_{-1}^{1} \tilde{f}\left(\widetilde{\mathbf{x}}, \kappa_{z}, \omega ; \widetilde{\mathbf{x}}_{i}\right)\left|J^{j}(\xi)\right| d \xi \\
& \quad=\int_{-1}^{\xi_{p}} \widetilde{f}\left(\widetilde{\mathbf{x}}, \kappa_{z}, \omega ; \widetilde{\mathbf{x}}_{i}\right)\left|J^{j}(\xi)\right| d \xi+\int_{\xi_{p}}^{1} \widetilde{f}\left(\widetilde{\mathbf{x}}, \kappa_{z}, \omega ; \widetilde{\mathbf{x}}_{i}\right)\left|J^{j}(\xi)\right| d \xi
\end{aligned}
$$

with $\xi_{p}$ being the natural coordinate of the collocation point $\widetilde{\mathbf{x}}_{i}, \widetilde{f}\left(\widetilde{\mathbf{x}}, \kappa_{z}, \omega ; \widetilde{\mathbf{x}}_{i}\right)$ the kernel of integrals in eqns (5) and (6), and $\left|J^{j}(\xi)\right|$ the Jacobian of the transformation to the natural coordinate system for element $\Sigma^{j}$. These integrals are numerically evaluated using a smooth polynomial transformation as described in Reference [14]. Accurate results are given in the next section for a number of integration points $10(p+1)$.

Finally, the boundary element matrices are assembled into a single system of equations relating sound pressure and particle normal displacement at nodal points:

$$
\widetilde{\mathbf{H}}\left(\widetilde{\mathbf{x}}, \kappa_{z}, \omega\right) \widetilde{p}\left(\widetilde{\mathbf{x}}, \kappa_{z}, \omega\right)=\widetilde{\mathbf{G}}\left(\widetilde{\mathbf{x}}, \kappa_{z}, \omega\right) \widetilde{u}\left(\widetilde{\mathbf{x}}, \kappa_{z}, \omega\right),
$$

where $\widetilde{p}\left(\widetilde{x}, \kappa_{z}, \omega\right)$ and $\widetilde{u}\left(\widetilde{\mathbf{x}}, \kappa_{z}, \omega\right)$ collect the nodal pressure and the normal displacement to the boundary, and $\widetilde{\mathbf{H}}\left(\widetilde{\mathbf{x}}, \kappa_{z}, \omega\right)$ and $\widetilde{\mathbf{G}}\left(\widetilde{\mathbf{x}}, \kappa_{z}, \omega\right)$ are the fully populated non-symmetrical boundary element system matrices.

\subsection{Spectral finite element method (SFEM)}

The spectral finite element formulation is based on the virtual work principle in the frequencywavenumber domain, which states [15]:

$$
\begin{gathered}
-\omega^{2} \int_{\Omega_{s}} \delta \widehat{\mathbf{u}}^{T}\left(\mathbf{x}, \kappa_{z}, \omega\right) \rho_{s} \widehat{\mathbf{u}}\left(\mathbf{x}, \kappa_{z}, \omega\right) d \Omega+\int_{\Omega_{s}} \delta \widehat{\boldsymbol{\varepsilon}}^{T}\left(\mathbf{x}, \kappa_{z}, \omega\right) \widehat{\boldsymbol{\sigma}}\left(\mathbf{x}, \kappa_{z}, \omega\right) d \Omega \\
=\int_{\Omega_{s}} \delta \widehat{\mathbf{u}}^{T}\left(\mathbf{x}, \kappa_{z}, \omega\right) \rho_{s} \widehat{\mathbf{b}}\left(\mathbf{x}, \kappa_{z}, \omega\right) d \Omega+\int_{\Gamma} \delta \widehat{\mathbf{u}}^{T}\left(\mathbf{x}, \kappa_{z}, \omega\right) \widehat{\mathbf{q}}\left(\mathbf{x}, \kappa_{z}, \omega\right) d \Gamma
\end{gathered}
$$

where $\widehat{\mathbf{u}}\left(\mathbf{x}, \kappa_{z}, \omega\right)$ is the displacement vector, $\widehat{\boldsymbol{\varepsilon}}\left(\mathbf{x}, \kappa_{z}, \omega\right)$ and $\widehat{\boldsymbol{\sigma}}\left(\mathbf{x}, \kappa_{z}, \omega\right)$ are respectively the strain and stress tensors, $\rho_{s} \widehat{\mathbf{b}}\left(\mathbf{x}, \kappa_{z}, \omega\right)$ is the body force in the domain $\Omega_{s}, \rho_{s}$ is the solid density, and $\widehat{\mathbf{q}}\left(\mathbf{x}, \kappa_{z}, \omega\right)$ is the nodal force vector. A variable preceded by $\delta$ denotes a compatible variation of the displacement or the strain field.

The stress tensor is related to the strain tensor through the constitutive relation $\widehat{\sigma}=\mathbf{C} \widehat{\varepsilon}$, where, in the case of a linear isotropic material, the constitutive matrix $\mathbf{C}$ depends on the Young's modulus $E$ and the Poisson ratio $\nu$.

The dependence of the strain tensor on the longitudinal coordinate $z$ can be removed considering the displacement definition in the frequency-wavenumber domain according to eqn (1):

$$
\widehat{\mathbf{u}}\left(\mathbf{x}, \kappa_{z}, \omega\right)=\widetilde{\mathbf{u}}\left(\widetilde{\mathbf{x}}, \kappa_{z}, \omega\right) e^{-\iota \kappa_{z} z} .
$$

Then, the strain tensor can be derived from the displacement vector $\widehat{\mathbf{u}}$ as:

$$
\widehat{\varepsilon}=\mathbf{L}_{1} \widehat{\mathbf{u}}+\mathbf{L}_{2} \frac{\partial \widehat{\mathbf{u}}}{\partial z}=\mathbf{L}_{1} \widehat{\mathbf{u}}-\iota \kappa_{z} \mathbf{L}_{2} \widehat{\mathbf{u}}
$$

where the differential operators are: 


$$
\mathbf{L}_{1}=\left[\begin{array}{ccc}
\frac{\partial}{\partial x} & 0 & 0 \\
0 & \frac{\partial}{\partial y} & 0 \\
0 & 0 & 0 \\
\frac{\partial}{\partial y} & \frac{\partial}{\partial x} & 0 \\
0 & 0 & \frac{\partial}{\partial y} \\
0 & 0 & \frac{\partial}{\partial x}
\end{array}\right], \quad \mathbf{L}_{2}=\left[\begin{array}{ccc}
0 & 0 & 0 \\
0 & 0 & 0 \\
0 & 0 & 1 \\
0 & 0 & 0 \\
0 & 1 & 0 \\
1 & 0 & 0
\end{array}\right]
$$

The 2.5D formulation is derived assuming that solid material properties and geometry are both homogeneous in the longitudinal direction. Therefore, eqn (9) is further elaborated by eliminating the dependency on the coordinate $z$ rewriting the volume integrals over the section $A_{s}$ of the domain integration $\Omega_{s}$ by the coordinate plane $z=0$ :

$$
-\omega^{2} \int_{A_{s}} \delta \widetilde{\mathbf{u}}^{T} \rho_{s} \widetilde{\mathbf{u}} d A+\int_{A_{s}} \delta \widetilde{\boldsymbol{\varepsilon}}^{T} \widetilde{\boldsymbol{\sigma}} d A=\int_{A_{s}} \delta \widetilde{\mathbf{u}}^{T} \rho_{s} \widetilde{\mathbf{b}} d A+\int_{\Sigma} \delta \widetilde{\mathbf{u}}^{T} \widetilde{\mathbf{q}} d \Sigma,
$$

where $\Sigma$ is defined as the boundary $\Gamma$ at $z=0$.

The solid subdomain is discretised into elements, where the approximated displacement vector $\widetilde{\mathbf{u}}$ is defined as:

$$
\widetilde{\mathbf{u}}=\varphi \widetilde{\mathbf{u}}^{e},
$$

where $\varphi$ are the two-dimensional shape functions obtained from the one-dimensional function $\phi(\xi)$ as:

$$
\varphi^{k}(\xi, \eta)=\phi^{i}(\xi) \phi^{j}(\eta), \quad k=(i-1)(p+1)+j .
$$

The proposed spectral finite element is defined from local nodal coordinates $(\xi, \eta) \in$ $([-1,1] \times[-1,1])$ at the LGL points.

Once the element approximation is defined, the strain tensor is expressed in terms of the nodal displacement as:

$$
\widetilde{\varepsilon}^{e}=\mathbf{L}_{1} \varphi \widetilde{\mathbf{u}}^{e}-\iota \kappa_{z} \mathbf{L}_{2} \varphi \widetilde{\mathbf{u}}^{e}=\mathbf{B}_{1} \widetilde{\mathbf{u}}^{e}-\iota \kappa_{z} \mathbf{B}_{2} \widetilde{\mathbf{u}}^{e},
$$

where $\mathbf{B}_{1}=\mathbf{L}_{1} \boldsymbol{\varphi}$ and $\mathbf{B}_{2}=\mathbf{L}_{2} \varphi$.

Introducing eqns (14) and (16) into eqn (13) and operating, the virtual work principle can be rewritten for each element as:

$$
\begin{aligned}
& -\omega^{2} \int_{A_{s}^{e}} \delta \widetilde{\mathbf{u}}^{e T} \varphi^{T} \rho_{s} \varphi \widetilde{\mathbf{u}}^{e} d A+\int_{A_{s}^{e}} \delta \widetilde{\mathbf{u}}^{e T} \mathbf{B}_{1}^{T} \mathbf{C} \mathbf{B}_{1} \widetilde{\mathbf{u}}^{e} d A \\
& -\iota \kappa_{z} \int_{A_{s}^{e}} \delta \widetilde{\mathbf{u}}^{e T}\left(\mathbf{B}_{1}^{T} \mathbf{C} \mathbf{B}_{2}+\mathbf{B}_{2}^{T} \mathbf{C} \mathbf{B}_{1}\right) \widetilde{\mathbf{u}}^{e} d A-\kappa_{z}^{2} \int_{A_{s}^{e}} \delta \widetilde{\mathbf{u}}^{e T} \mathbf{B}_{2}^{T} \mathbf{C} \mathbf{B}_{2} \widetilde{\mathbf{u}}^{e} d A \\
& =\int_{A_{s}^{e}} \delta \widetilde{\mathbf{u}}^{e T} \boldsymbol{\varphi}^{T} \rho_{s} \widetilde{\mathbf{b}}^{e} d A+\int_{\Sigma^{e}} \delta \widetilde{\mathbf{u}}^{e T} \boldsymbol{\varphi}^{T} \widetilde{\mathbf{q}}^{e} d \Sigma,
\end{aligned}
$$

where $A_{s}^{e}$ is the cross-sectional area of the three dimensional element in the $z$ direction and $\Sigma^{e}$ its boundary. The following expression is obtained taking into account that eqn (17) must be satisfied for any compatible displacement $\delta \widetilde{\mathbf{u}}^{e}$ :

$$
\left[-\omega^{2} \mathbf{M}^{e}+\mathbf{K}_{0}^{e}-\iota \kappa_{z} \mathbf{K}_{1}^{e}-\kappa_{z}^{2} \mathbf{K}_{2}^{e}\right] \widetilde{\mathbf{u}}^{e}=\widetilde{\mathbf{f}}^{e}
$$


where $\mathbf{M}^{e}$ is the element mass matrix, $\mathbf{K}_{0}^{e}, \mathbf{K}_{1}^{e}$ and $\mathbf{K}_{2}^{e}$ are the stiffness element matrices and $\widehat{\mathbf{f}}^{e}$ is a vector that collects the forces applied to the element. These matrices are defined by:

$$
\begin{aligned}
\mathbf{M}^{e} & =\int_{A_{s}^{e}} \varphi^{T} \rho_{s} \varphi d A, \\
\mathbf{K}_{0}^{e} & =\int_{A_{s}^{e}} \mathbf{B}_{1}^{T} \mathbf{C} \mathbf{B}_{1} d A, \\
\mathbf{K}_{1}^{e} & =\int_{A_{s}^{e}}\left(\mathbf{B}_{1}^{T} \mathbf{C B}_{2}-\mathbf{B}_{2}^{T} \mathbf{C B}_{1}\right) d A, \\
\mathbf{K}_{2}^{e} & =\int_{A_{s}^{e}} \mathbf{B}_{2}^{T} \mathbf{C B}_{2} d A,
\end{aligned}
$$

and the external load vector $\widetilde{\mathbf{f}}^{e}$ as:

$$
\widetilde{\mathbf{f}}^{e}\left(\widetilde{\mathbf{x}}, \kappa_{z}, \omega\right)=\int_{A_{s}^{e}} \varphi^{T} \rho_{s} \widetilde{\mathbf{b}}^{e}\left(\widetilde{\mathbf{x}}, \kappa_{z}, \omega\right) d A+\int_{\Sigma^{e}} \boldsymbol{\varphi}^{T} \widetilde{\mathbf{q}}^{e}\left(\widetilde{\mathbf{x}}, \kappa_{z}, \omega\right) d \Sigma,
$$

which contains the contributions of both body forces and surface loads.

Then, eqn (18) can be written as:

$$
\widetilde{\mathbf{K}} \widetilde{\mathbf{u}}=\widetilde{\mathbf{f}},
$$

where $\widetilde{\mathbf{K}}=\left[-\omega^{2} \mathbf{M}+\mathbf{K}_{0}-\iota \kappa_{z} \mathbf{K}_{1}-\kappa_{z}^{2} \mathbf{K}_{2}\right]$ is the dynamic stiffness matrix obtained in the frequency-wavenumber domain from the assembled mass $\mathbf{M}$ matrix, and stiffness $\mathbf{K}_{0}, \mathbf{K}_{1}$ and $\mathbf{K}_{2}$ matrices.

The mass $\mathbf{M}^{e}$ matrix and stiffness $\mathbf{K}_{0}^{e}, \mathbf{K}_{1}^{e}$ and $\mathbf{K}_{2}^{e}$ matrices are numerically integrated using a LGL quadrature of order $p$. Therefore, the nodal element coordinates coincide with the element integration points producing a diagonal mass $\mathbf{M}^{e}$ matrix and stiffness $\mathbf{K}_{2}^{e}$ matrices.

\subsection{SBEM-SFEM coupling}

Eqns (8) and (24) are coupled by imposing equilibrium of forces, compatibility of normal displacement and null shear stresses at the interface $\Gamma$. These equations are assembled into a single comprehensive system, together with the equilibrium and compatibility conditions.

The load vector $\widetilde{\mathbf{f}}_{f}$ at the fluid-solid interface is obtained by integrating the fluid pressure field $\widetilde{p}_{f}$ over the boundary $\Gamma$ :

$$
\widetilde{\mathbf{f}}_{f}=-\int_{\Gamma} \varphi^{T} \mathbf{n} \phi \widetilde{p}_{f} d \Gamma=-\mathbf{R}^{T} \widetilde{p}_{f}
$$

where $\mathbf{n}$ is the outward normal vector along $\Gamma$, and $\mathbf{R}$ is the coupling fluid-solid matrix which relates force at the solid subdomain and pressure at the boundary. Substituting eqn (25) into eqn (24) yields:

$$
\left[\begin{array}{ll}
\widetilde{\mathbf{K}}_{s s} & \widetilde{\mathbf{K}}_{s f} \\
\widetilde{\mathbf{K}}_{f s} & \widetilde{\mathbf{K}}_{f f}
\end{array}\right]\left[\begin{array}{c}
\widetilde{\mathbf{u}}_{s} \\
\widetilde{\mathbf{u}}_{f}
\end{array}\right]=\left[\begin{array}{c}
\widetilde{\mathbf{f}}_{s} \\
-\mathbf{R}^{T} \widetilde{p}_{f}
\end{array}\right],
$$

where subscript $f$ indicates degrees of freedom belonging to $\Gamma$ and $s$ stands for the rest of solid degrees of freedom.

Finally, the coupling of eqns (8) and (26) is carried out with the imposition of compatibility of normal displacement at the boundary $\Gamma$, and null shear-stresses. Both 


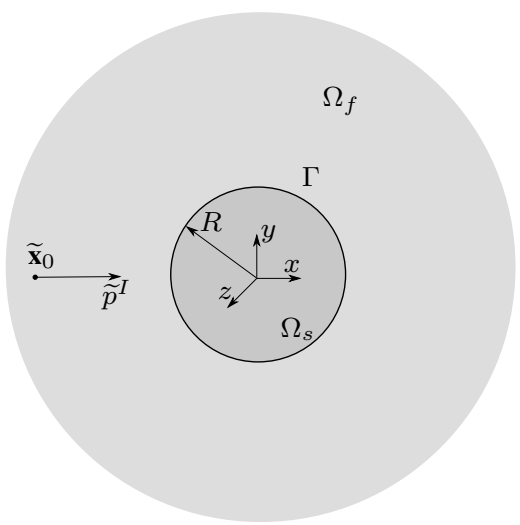

Figure 2: Elastice inchusion in an unbounded acoustic region.

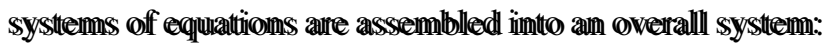

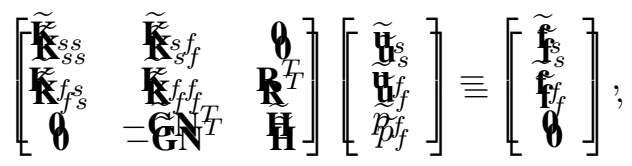

where $\mathbf{N}$ is a matrix containing the outward normal vector $\mathbf{n}$ at the boundary. Eqn (27) is

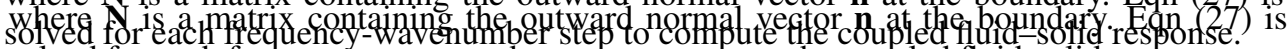
solved for each frequency-wavenumber step to compute the coupled fluid-solid response.

\section{MODEL VERIFICATION}

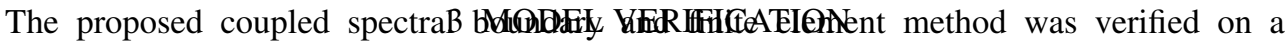

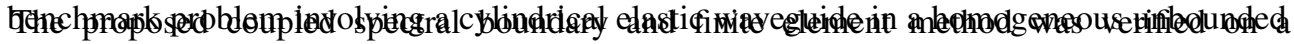

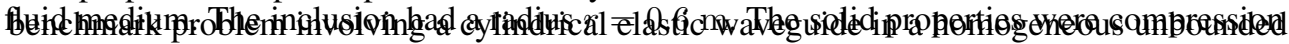

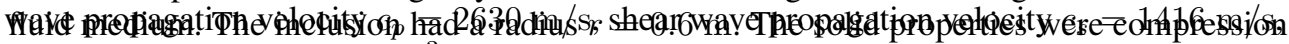

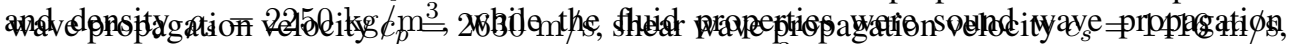

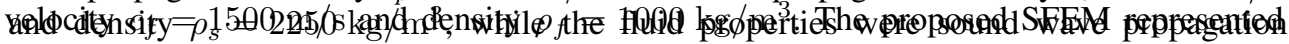

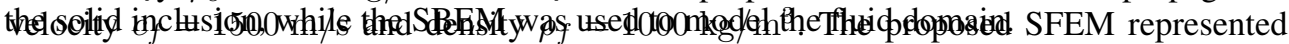

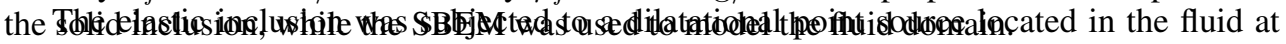

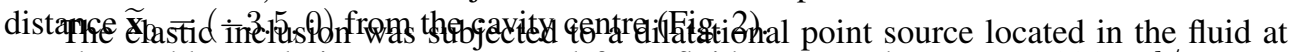

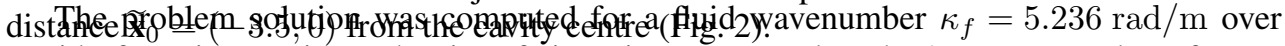

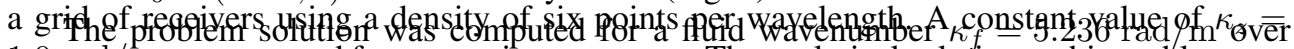

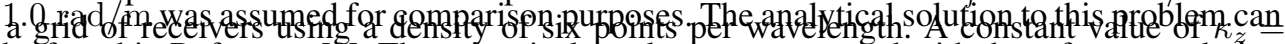

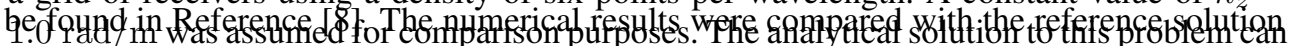

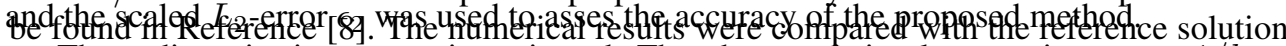

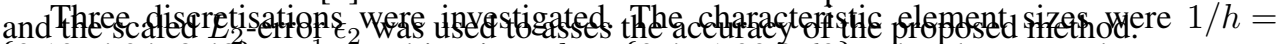

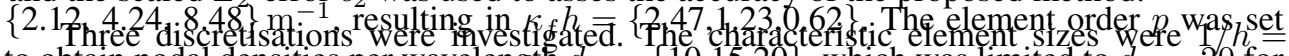

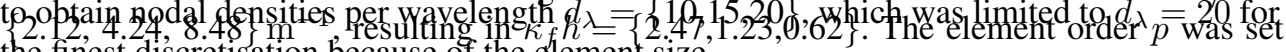
the finest discretisation because of the element size; $\$ 15,20\}$, which was limited to $d_{\lambda}=20$ for

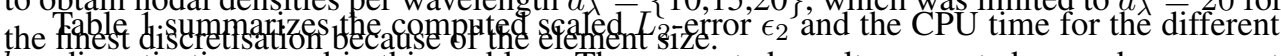
$h-p$ discretisations used in this problem. The computed results presented a good agreement with The le summarizes the computed scaled $L_{2}$-error $\epsilon_{2}$ and the CPU time

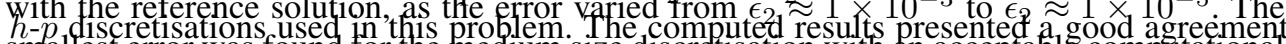
smallest error was found.for the nedium size discretisation with an afceptable computafiopal smallest error was found for the medium size discretisation with an acceptable computational

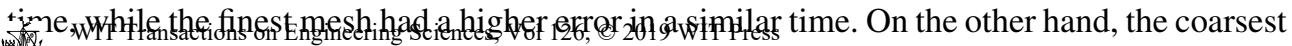

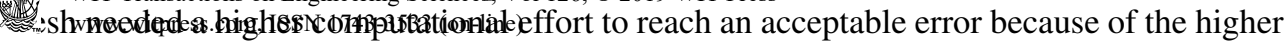
element order approximation. Therefore, a $h p$-refinement is more efficient in terms of error and computational effort than a single $h$-refinement or $p$-refinement. 
Table 1: Summary of scaled $L_{2}$-error $\epsilon_{2}$ of the total fluid pressure, horizontal and longitudinal solid displacement and CPU time, computed for the different $h p$ discretisations.

\begin{tabular}{ccccccc}
\hline $1 / h\left[\mathrm{~m}^{-1}\right]$ & $p$ & $d_{\lambda}$ & $\epsilon_{2}^{p}$ & $\epsilon_{2}^{u_{x}}$ & $\epsilon_{2}^{u_{z}}$ & CPU time $[\mathrm{s}]$ \\
\hline & 2 & 5 & $2.14 \times 10^{-1}$ & $2.24 \times 10^{-1}$ & $5.76 \times 10^{-2}$ & 0.526 \\
& 3 & 7.5 & $1.22 \times 10^{-2}$ & $1.58 \times 10^{-2}$ & $8.07 \times 10^{-3}$ & 1.051 \\
2.12 & 4 & 10 & $1.78 \times 10^{-3}$ & $2.08 \times 10^{-3}$ & $1.08 \times 10^{-3}$ & 1.713 \\
& 6 & 15 & $5.71 \times 10^{-4}$ & $4.90 \times 10^{-4}$ & $1.03 \times 10^{-3}$ & 4.379 \\
& 8 & 20 & $5.01 \times 10^{-4}$ & $3.99 \times 10^{-4}$ & $1.00 \times 10^{-4}$ & 11.36 \\
\hline \multirow{3}{*}{4.24} & 2 & 10 & $3.93 \times 10^{-3}$ & $5.50 \times 10^{-3}$ & $3.92 \times 10^{-3}$ & 0.899 \\
& 3 & 15 & $1.29 \times 10^{-4}$ & $3.08 \times 10^{-4}$ & $2.37 \times 10^{-4}$ & 2.018 \\
& 4 & 20 & $6.0 \times 10^{-5}$ & $2.75 \times 10^{-5}$ & $7.55 \times 10^{-5}$ & 3.976 \\
\hline 8.48 & 2 & 20 & $1.67 \times 10^{-4}$ & $4.20 \times 10^{-4}$ & $3.24 \times 10^{-4}$ & 2.449 \\
\hline
\end{tabular}

time, while the finest mesh had a higher error in a similar time. On the other hand, the coarsest mesh needed a higher computational effort to reach an acceptable error because of the higher element order approximation. Therefore, a $h p$-refinement is more efficient in terms of error and computational effort than a single $h$-refinement or $p$-refinement.

\section{NUMERICAL EXAMPLE}

Finally, the capabilities of the proposed method are explored with a numerical example. The problem solved herein was a study of the wave field scattered by an array of elastic cylinders submerged in an inviscid fluid medium (Fig. 3). The elastic cylinders had an external radius $r=0.4 \mathrm{~m}$ and a thickness $t=0.05 \mathrm{~m}$. The centres of the scatterers were equally spaced $s=1.2 \mathrm{~m}$. The material was assumed to be PVC, with compression wave propagation velocity $c_{p}=2143 \mathrm{~m} / \mathrm{s}$, shear wave propagation velocity $c_{s}=875 \mathrm{~m} / \mathrm{s}$, and density $\rho=1400 \mathrm{~kg} / \mathrm{m}^{3}$. The outer fluid was water, with sound wave propagation velocity $c_{f}=1500 \mathrm{~m} / \mathrm{s}$ and density $\rho_{f}=1000 \mathrm{~kg} / \mathrm{m}^{3}$. Three configurations were considered based on the interior of the scatterers: $i$ ) water-filled; $i i$ ) air-filled, with sound propagation velocity $340 \mathrm{~m} / \mathrm{s}$ and density $1.225 \mathrm{~kg} / \mathrm{m}^{3}$; and iii) state of vacuum.

The array of scatterers was subjected to a dilatational point source located in the fluid at position $\widetilde{\mathbf{x}}_{0}=(-4.0,0)$ (Fig. 3). The incident wave field $\widetilde{p}^{I}$ was defined as in the previous section. The problem response of the fluid-structure system was studied for a frequency $f=6400 \mathrm{~Hz}$.

The scatterers were modelled with spectral finite elements while outer and inner fluid subdomains were represented with the SBEM. The discretisation was chosen to enable a nodal density per wavelength of $d_{\lambda}=12$ in the outer fluid, while the scatterers and the inner fluid meshes were defined to match the outer fluid mesh. The outer fluid was modelled with 34 elements, so the cylinders and the inner fluid were modelled again with 34 elements. The element order was set to $p=4$ in all cases. This discretisation resulted in a nodal density $d_{\lambda}=12$ with and $\kappa_{f} h=2$.

The 3D solution was computed as the superposition of 2D problems with different longitudinal wavenumbers by means of eqn (2). The 3D solution can be computed in a set of regularly spaced points along the longitudinal direction where $\Delta z=2 \pi / \max \left\{\kappa_{z}\right\}$. The maximum wavenumber $\max \left\{\kappa_{z}\right\}=160 \mathrm{rad} / \mathrm{m}$ was chosen to compute the solution enabling six points in a longitudinal wavelength, $\Delta z=2 \pi / \max \left\{\kappa_{z}\right\}=0.0393 \mathrm{~m}$. The 


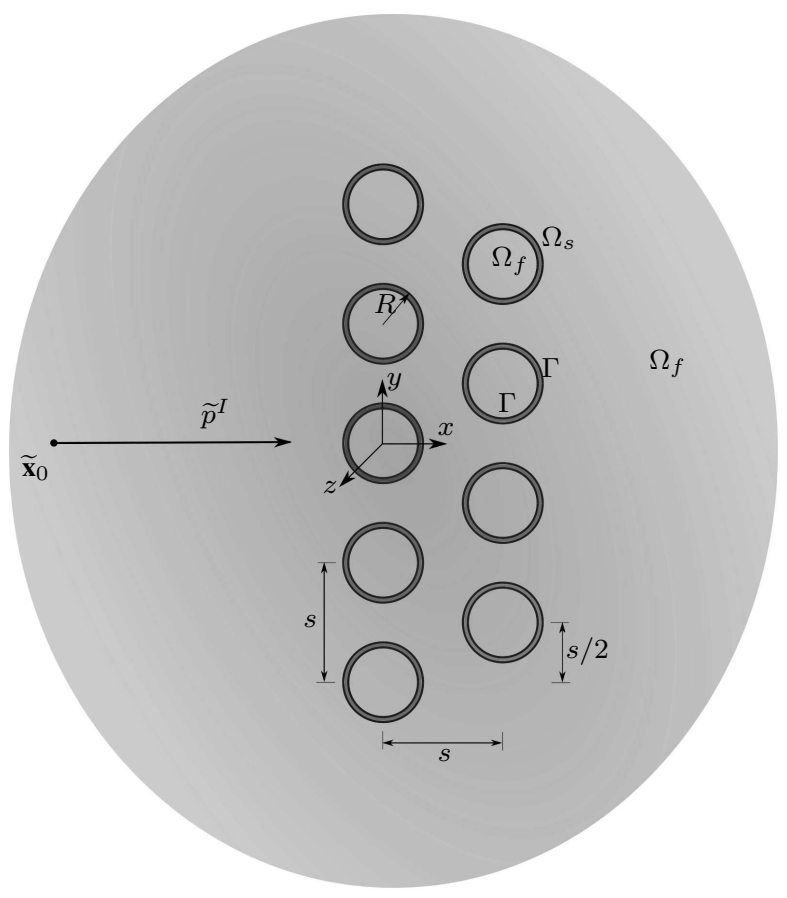

Figufe 3: Configufation of seatterers:

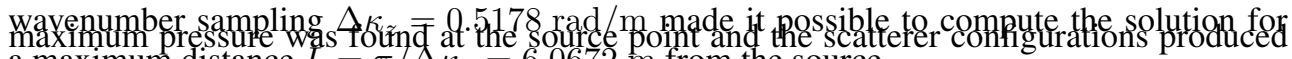

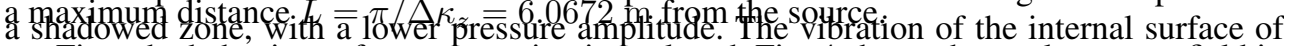

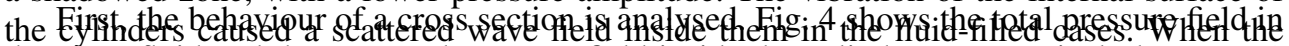

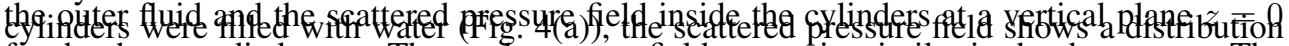

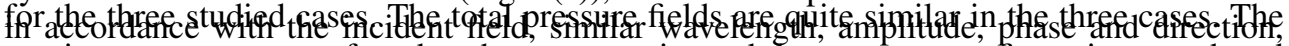

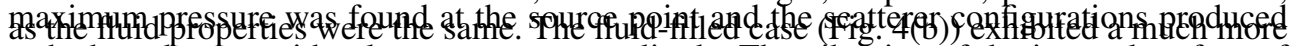

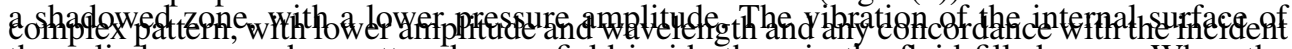

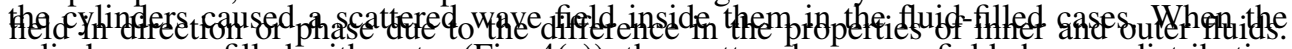

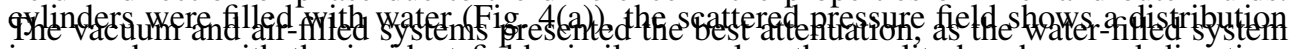

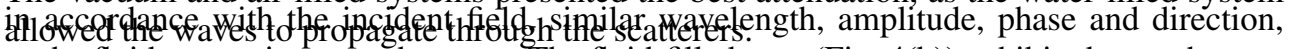
as the fluid properties were the same. The fluid-filled case (Fig. 4(b)) exhibited a much more

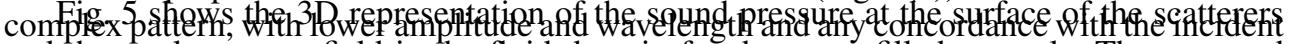

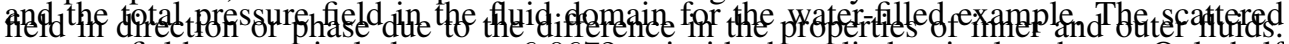

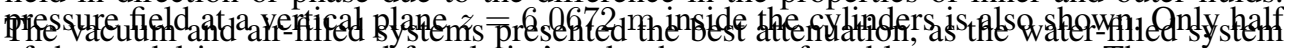

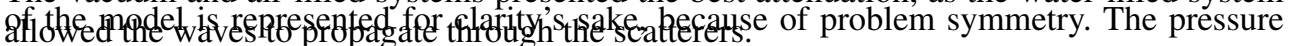
fielchad a spherical distribution centred at the source which was wrogressively decreasing as the distance to the source increased Fig. 6 shows the deformed shape of the sqlid scatterers external surface and displays the normal displacement to the bound ary in the colour sale. The displacement held also had spherical, distribution centered at the source which decreased

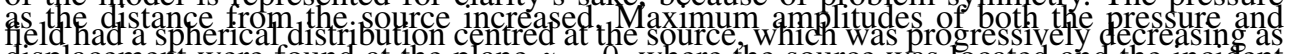

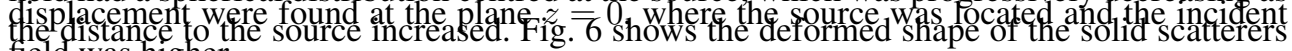
theld was higher.
external surface and displays the normal displacement to the boundary in the colour scale. The

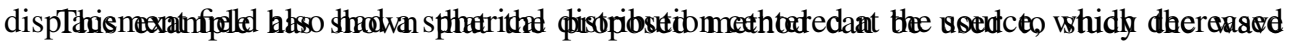
propagation in a fluid-filled elastic waveguides immersed in an unbounded acoustic medium. The proposed method was able to represent different fluid properties for the unbounded fluid

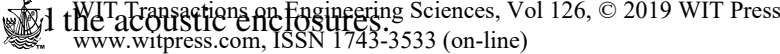




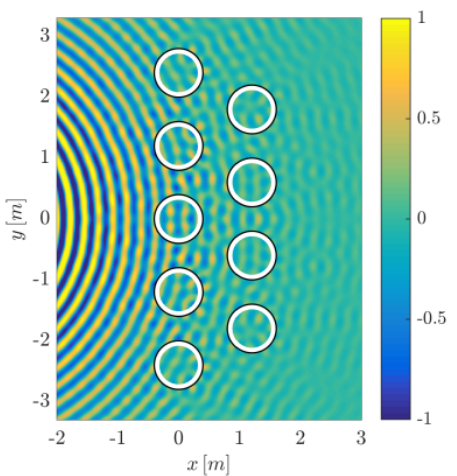

(a)

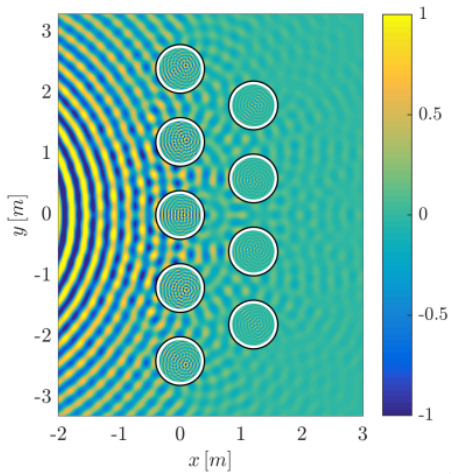

(b)

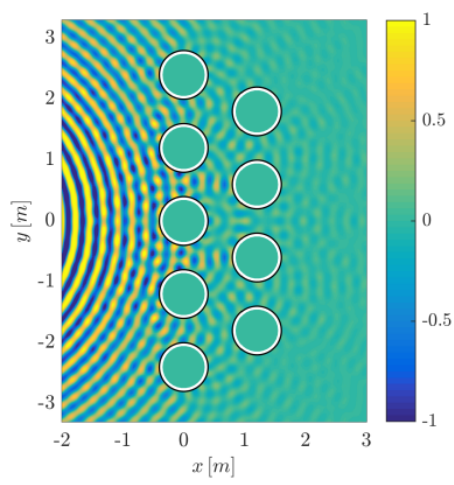

(c)

Figure 4: Real part of the pressure field at cross-section of the scatterers system at the vertical plane $z=0$ for the (a) water-filled cylinders, (b) air-filled cylinders and (c) vacuum cylinders. Sound pressure in water medium was normalised to $0.15 \mathrm{~Pa}$, and to $5 \times 10^{-5} \mathrm{~Pa}$ in air medium.

as the distance from the source increased. Maximum amplitudes of both the pressure and displacement were found at the plane $z=0$, where the source was located and the incident field was higher.

This example has shown that the proposed method can be used to study the wave propagation in a fluid-filled elastic waveguides immersed in an unbounded acoustic medium. The proposed method was able to represent different fluid properties for the unbounded fluid and the acoustic enclosures.

\section{CONCLUSIONS}

This work has proposed a spectral element formulation based on the BEM and the FEM to study fluid and solid wave propagation. The presented methodology looks at 3D problems whose materials and geometric properties remain homogeneous in one direction. Solid subdomains were modelled with the SFEM, whereas the unbounded fluid media and acoustic enclosures were represented by the SBEM. The coupling of both methods was carried out by 


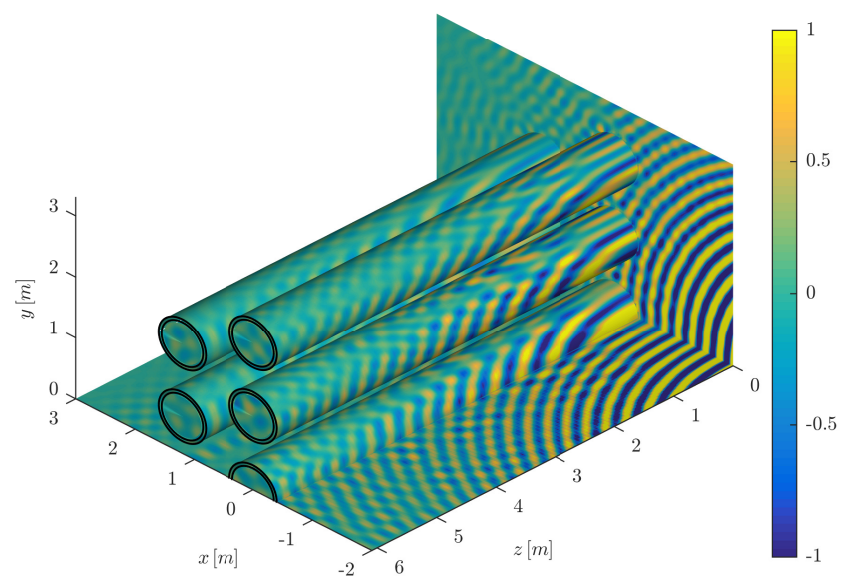

Figure 5: Real part of the pressure field (normalised to $0.15 \mathrm{~Pa}$ ) in an unbounded fluid by a system of water-filled elastic scatterers.

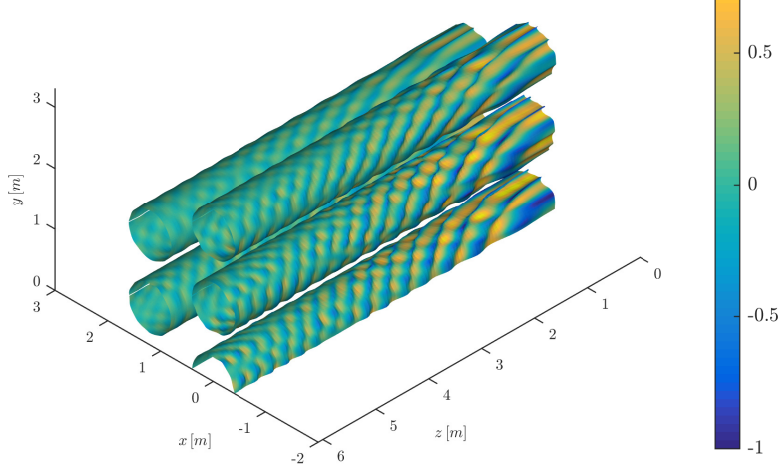

Figure 6: Real part of normal displacement at external surface (normalised to $1.2 \times$ $10^{-12} \mathrm{~m}$ ) in a system of water-filled elastic scatterers, represented over the deformed shape.

imposing the appropriate boundary conditions at the interface to study fluid-structure wave propagation.

The model was verified with a benchmark problem having an analytical solution. The coupled SBEM-SFEM was verified with a problem concerning a cylindrical elastic waveguide in an unbounded fluid medium. Numerical results show good agreement with the analytical solution. An $h-p$ analysis shows that an optimal solution in terms of accuracy and computational effort can be obtained through an $h p$-refinement. Discretisations with a nodal density per wavelength of ten are enough to achieve reasonable accuracy. The accuracy improves by increasing the nodal density per wavelength up to twenty. 
Finally, the coupled methodology was used to study the wave field radiated by a three dimensional fluid-filled elastic scatterers system submerged in an unbounded acoustic medium. The sound pressure field and the scatterers' surface displacements were studied. The proposed method made it possible to represent different fluids for the unbounded medium and the acoustic enclosures.

\section{ACKNOWLEDGEMENTS}

The research work presented herein was supported by the Spanish Ministry of the Economy and Competitiveness (Ministerio de Economía y Competitividad) through research project BIA2016-75042-C2-1-R. It was also supported by project POCI-01-0247-FEDER-017759 (SmartCore - Desenvolvimento de painéis multifuncionais de elevada performance), funded by the Operational Program for Competitiveness and Internationalization (POCI) of Portugal 2020, with the support of the European Regional Development Fund (FEDER). Financial support is gratefully acknowledged. The authors also wish to acknowledge the support provided by the Andalusian Scientific Computing Centre (CICA).

\section{REFERENCES}

[1] Babuška, I., Ihlenburg, F., Paik, E.T. \& Sauter, S.A., A generalized finite element method for solving the Helmholtz equation in two dimensions with minimal pollution. Computer Methods in Applied Mechanics and Engineering, 128(3), pp. 325-359, 1995.

[2] Ihlenburg, F., Babuška, I. \& Sauter, S., Reliability of finite element methods for the numerical computation of waves. Advances in Engineering Software, 28(7), pp. 417424, 1997.

[3] Oberai, A.A. \& Pinsky, P.M., A numerical comparison of finite element methods for the Helmholtz equation. Journal of Computational Acoustics, 08(01), pp. 211-221, 2000.

[4] Willberg, C., Duczek, S., Vivar Perez, J.M., Schmicker, D. \& Gabbert, U., Comparison of different higher order finite element schemes for the simulation of Lamb waves. Computer Methods in Applied Mechanics and Engineering, 241-244, pp. 246-261, 2012.

[5] Patera, A.T., A spectral element method for fluid dynamics: Laminar flow in a channel expansion. Journal of Computational Physics, 54(3), pp. 468-488, 1984.

[6] Ostachowicz, W., Kudela, P., Krawczuk, M. \& Zak, A., Guided Waves in Structures for SHM: The Time-domain Spectral Element Method, Wiley, 2012.

[7] Cerrato, A., Rodríguez-Tembleque, L., González, J.A. \& Ferri Aliabadi, M.H., A coupled finite and boundary spectral element method for linear water-wave propagation problems. Applied Mathematical Modelling, 48, pp. 1-20, 2017.

[8] Pereira, A., Tadeu, A. \& António, J., Influence of the cross-section geometry of a cylindrical solid submerged in an acoustic medium on wave propagation. Wave Motion, 36(1), pp. 23-39, 2002.

[9] Gavrić, L., Computation of propagative waves in free rail using a finite element technique. Journal of Sound and Vibration, 185(3), pp. 531-543, 1995.

[10] Gavrić, L., Finite element computation of dispersion properties of thin-walled waveguides. Journal of Sound and Vibration, 173(1), pp. 113-124, 1994.

[11] Domínguez, J., Boundary elements in dynamics, Computational Mechanics Publications and Elsevier Applied Science, 1993.

[12] Tadeu, A. \& Godinho, L., Three-dimensional wave scattering by a fixed cylindrical inclusion submerged in a fluid medium. Engineering Analysis with Boundary Elements, 23(9), pp. 745-755, 1999. 
[13] Fornberg, B. \& Zuev, J., The Runge phenomenon and spatially variable shape parameters in RBF interpolation. Computers and Mathematics with Applications, 54(3), pp. 379-398, 2007.

[14] Wu, T.W., Boundary Element Acoustics Fundamentals and Computer Codes, WIT Press, 2000.

[15] Zienkiewicz, O., The Finite Element Method, 3rd edn, McGraw-Hill, 1986. 